\title{
GPS navigation data bit decoding error during strong equatorial scintillation
}

\author{
Dongyang $\mathrm{Xu}^{1}$ (D) Y.T. Jade Morton ${ }^{2}$
}

Received: 3 June 2018 / Accepted: 11 August 2018 / Published online: 17 August 2018

(c) The Author(s) 2018

\begin{abstract}
Strong equatorial scintillation is often characterized by simultaneous fast phase changes and deep amplitude fading. The combined effect poses a challenge for GNSS receiver carrier tracking performance. One of the consequences of the strong scintillation is increased navigation message data bit decoding error. Understanding the rate of the data bit decoding error under equatorial scintillation is essential for high accuracy and high integrity applications. We present the statistical relationship between the data bit decoding error occurrences and the intensity of amplitude scintillation based on the processing of intermediate frequency GPS scintillation data collected on Ascension Island in March 2013. A third-order phase lock loop (PLL) is implemented to process the data and to access the data bit error typically expected in conventional receivers. A Kalman filter-based PLL is also used to process the same data to demonstrate that the data bit decoding error can be reduced through advanced carrier tracking designs.
\end{abstract}

Keywords GNSS navigation data bit decoding · Equatorial scintillation · GNSS carrier tracking loop

\section{Introduction}

The ionospheric scintillation phenomenon refers to the random amplitude and phase fluctuations observed on radio signals propagating through plasma irregularities in the ionosphere (Yeh and Liu 1982). Ionospheric scintillation most frequently occurs in equatorial and high latitude areas, while the strongest effects are often observed in equatorial areas characterized by the simultaneous occurrence of fast phase changes and deep amplitude fading (Basu et al. 2002; Morton et al. 2015a, b). Strong equatorial scintillation is known to degrade receiver carrier tracking performance, causing measurement error, cycle slips, and even loss of lock signals (Kintner et al. 2007; Seo et al. 2009). In the real data analysis presented in our previous publications (Jiao et al. 2016; Myer and Morton 2018), cycle slips and loss of lock are frequently observed in commercial receivers in the equatorial region, some of which can last more than $2 \mathrm{~h}$.

Dongyang Xu

dyxu@rams.colostate.edu

1 Department of Electrical and Computer Engineering, Colorado State University, Fort Collins, CO 80523, USA

2 University of Colorado at Boulder, Boulder, CO, USA
The carrier tracking loop performance degradation caused by ionospheric scintillation impacts a variety of GNSS applications. For example, Xiong et al. (2016) and Buchert et al. (2015) reported incidences of the Swarm satellites (operated by the European Space Agency) failing to generate position solutions due to loss of GPS signals over equatorial areas where ionospheric plasma irregularities are known to occur frequently. For high accuracy applications, ionospheric scintillation causes errors or loss of measurements in differential GPS (DGPS) and real-time kinematic (RTK) solutions (Aquino et al. 2005; Morton 2014). Similarly, ionospheric scintillation affects Satellite-Based Augmentation System (SBAS) which directly impacts applications such as aviation and precision agriculture (Conker et al. 2003; Lee et al. 2017).

A pre-requisite to developing GNSS receiver processing algorithms that can mitigate the adverse effects the ionospheric scintillation has on GNSS applications is understanding of the characteristics of the effects. In recent years, the availability of massive scintillation measurement data has led to numerous studies focused on the temporal and spectral characteristics of the scintillation signal amplitude and phase (Seo et al. 2009, 2011; Oliveira et al. 2014; Jiao et al. 2016). This work will focus on the navigation 
message bit decoding error (BDE) during strong equatorial scintillation.

The navigation message being broadcast by GPS satellites contains information on satellite orbital and timing information needed to compute position, velocity, and time (PVT) solutions, as well as correction parameters to improve the solutions' accuracy. During equatorial scintillation, it is known that there is increased navigation data BDE which degrades the accuracy of the receiver's navigation solutions, especially when multiple satellites experience strong scintillation (Carroll and Morton 2014; Zhang et al. 2010). However, the quantitative dependence of the BDE on the scintillation level has not been studied. The main objective of this work is to present a quantitative analysis of the BDE during strong equatorial scintillation based on processing results from real equatorial scintillation data. Such analysis is necessary because the degraded decoding performance can negatively impact high accuracy applications and differential systems where rover receivers and their corresponding ground correction networks share the same satellite orbital and timing parameters.

The data used in this study were collected on Ascension Island during strong scintillation on March 7-10, 2013. The data collection system consists of an array of softwaredefined radios (SDR) which samples multi-GNSS IF signals in the event of scintillation. A network of these event-driven data collection systems has been established by the author's group since 2009 (Morton et al. 2015a, b). The analysis conducted here is based on about $14 \mathrm{~h}$ of GPS L1 intermediate frequency samples captured on six satellites during scintillation periods with the $S_{4}$ index over 0.5 .

Two carrier tracking algorithms are used to process the data. The first is a conventional third-order phase lock loop (PLL) carrier tracking algorithm (Ward et al. 2005; Tsui 2005). There are two reasons for using this algorithm to process the data: to assess the BDE typically experienced by commercial receivers in the field and to establish correlations between the BDE distributions and the amplitude scintillation and fading characteristics such as fading level and duration.

In addition, an advanced adaptive Kalman filter-based PLL (AKF-PLL) discussed in (Xu et al. 2017) is also implemented. The AKF-PLL architecture is adopted from the general state-based framework presented in (Yang et al. 2017a, b) while incorporating phase scintillation in its process noise model. A subset of the scintillation data is used as test data to compare these two algorithms to assess the improvement of the advanced AKF-based PLL over the conventional PLL on the BDE performance.

Following the descriptions of the algorithms and the data used in this work, we discuss the statistical correlations between the BDE occurrence and the level of amplitude scintillation and fading. Then, a comparison of the BDE performance using different carrier tracking algorithms will be presented. Finally, an overall summary is given.

\section{Algorithm's description}

Figure 1 shows the receiver carrier tracking architecture. It consists of correlators, a carrier PLL, a code tracking loop, and navigation data bit recording. The code tracking loop is a second-order delay lock loop (DLL) with no carrier aiding due to the stationary receiver platform. A normalized earlyminus-late envelope discriminator and a loop filter with a noise bandwidth of $0.25 \mathrm{~Hz}$ are implemented in the DLL.

Two PLLs were implemented in this project: a conventional proportion-integration filter-based PLL (PIF-PLL) and an AKF-PLL. Both PIF-PLL and AKF-PLL are well documented in the previous literature (Ward et al. 2005; Tsui 2005; Xu et al. 2017; Yang et al. 2017a, b). Note that the objective of this project is not the development of new
Fig. 1 SDR receiver and BDE assessment architecture used in this study

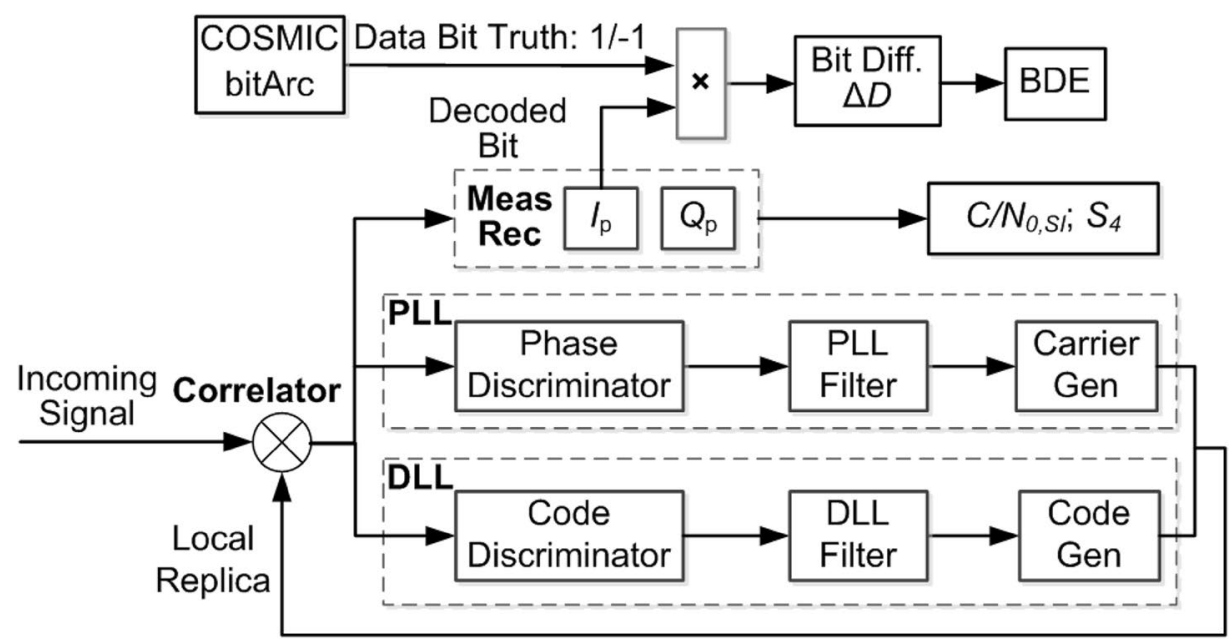


carrier tracking algorithms. Instead, it is the assessment of BDE performances of existing techniques. Therefore, only brief descriptions are given below for the sake of completeness, followed by scintillation intensity indicators and bit error estimation.

\section{PIF-PLL and AKF-PLL implementations}

The PIF-PLL transfer function is defined by two parameters: its equivalent noise bandwidth and the integration time (Ward et al. 2005). In practical implementations, they are empirically determined as a compromise between the practical requirements on the receiver dynamic performance and noise performance. In (Xu et al. 2014), several PIF-PLL bandwidth values ranging from 2 to $15 \mathrm{~Hz}$ are used to track the same real scintillation data collected on Ascension Island to evaluate the effect of different bandwidths for scintillation tracking. Based on the tracking results of $5 \mathrm{~h}$ of strong scintillation signals, the $2 \mathrm{~Hz}$ bandwidth implementation generated the least number of signal loss-of-lock incidences. Such a low PIF-PLL bandwidth is a possible option because the receiver front end was driven by a low phase noise ovencontrolled crystal oscillator (OCXO). In this study, the same $2 \mathrm{~Hz}$ bandwidth was applied to the PIF implementations, which successfully maintained lock of the scintillation signals throughout the selected scintillation data.

The AKF-PLL is implemented to demonstrate the potential to improve $\mathrm{BDE}$ during strong scintillation. A unique feature of the AKF-PLL implementation is the incorporation of phase scintillation effects in its system noise model. This is achieved by treating the phase scintillation as an equivalent oscillator noise model which can be described by the Allan variance parameters. System noise due to the actual receiver oscillator and platform dynamics are also included. Its measurement noise model is based on the thermal noise and can be updated according to real-time estimated $C / N_{0}$. The steady-state Kalman gain is numerically solved based on the system model and the measurement model at each iteration of the tracking loop operation. The AKF-PLL implementation has been demonstrated to be robust in tracking scintillation signals on airborne platforms. For mathematical representations of the models and implementation details, please refer to Xu et al. (2017) and Yang et al. (2017a, b).

For both PIF-PLL and AKF-PLL implementations, the integration time was $10 \mathrm{~ms}$ as a previous study found that it yields better tracking results in strong equatorial scintillations (Psiaki et al. 2007).

\section{Scintillation intensity indicator calculation}

The prompt channel correlator outputs $\left(I_{\mathrm{p}}, Q_{\mathrm{p}}\right)$ are used to calculate the amplitude scintillation and fading indicators. The scintillation and fading indicators include the $S_{4}$ index and the high-rate carrier-to-noise ratio (denoted as $C / N_{0, \mathrm{SI}}$ ) first presented in Xu and Morton (2017). The $S_{4}$ index describes the magnitude of the scintillation signal amplitude fluctuation. The $C / N_{0, \text { SI }}$ is a quantity derived from the normalized signal intensity ( $\left.\mathrm{SI}_{\text {norm }}\right)$ and the nominal signal carrier-to-noise ratio $\left(C / N_{0, \text { nominal }}\right)$ with the purpose to capture the amplitude fading level and duration with a resolution of tens of milliseconds.

To calculate $S_{4}$ and $C / N_{0, S I}, \mathrm{SI}_{\text {norm }}$ needs to be obtained first. The $\mathrm{SI}_{\text {norm }}$ calculation is based on the method discussed in Van Dierendonck et al. (1993):

$\mathrm{WBP}_{k}=\sum_{20 *(k-1)+1}^{20 * k}\left[\left(I_{i}^{1}\right)^{2}+\left(Q_{i}^{1}\right)^{2}\right]$

$\mathrm{NBP}_{k}=\left(\sum_{20 *(k-1)+1}^{20 * k} I_{i}^{1}\right)^{2}+\left(\sum_{20 *(k-1)+1}^{20 * k} Q_{i}^{1}\right)^{2}$

$\mathrm{SI}_{\text {raw }, k}=\mathrm{NBP}_{k}-\mathrm{WBP}_{k}$

$\mathrm{SI}_{\text {norm }, k}=10 \times \log 10\left(\frac{\mathrm{SI}_{\text {raw }, k}}{\mathrm{SI}_{\text {trend }, k}}\right)$

where WBP and NBP denote wide band power and narrow band power, respectively. $I_{i}^{1}$ and $Q_{i}^{1}$ are the $1 \mathrm{~ms}$ correlator outputs between bit $k-1$ and $k$. $\mathrm{SI}_{\text {raw }}$ denotes the raw signal intensity. The normalized signal intensity $\mathrm{SI}_{\text {norm }}$ is then obtained by normalizing $\mathrm{SI}_{\text {raw }}$ with respect to its own low frequency trend $\mathrm{SI}_{\text {trend }}$. $\mathrm{SI}_{\text {trend }}$ can be estimated using a sixth order Butterworth filter (Van Dierendonck et al. 1993).

The $S_{4}$ index is also computed from the $\mathrm{SI}_{\text {norm }}$ estimate (Van Dierendonck et al. 1993):

$S_{4}=\sqrt{\frac{\left\langle\mathrm{SI}_{\text {norm }}^{2}\right\rangle-\left\langle\mathrm{SI}_{\text {norm }}\right\rangle^{2}}{\left\langle\mathrm{SI}_{\text {norm }}\right\rangle^{2}}}$

where $\langle\cdot\rangle$ represents the averaging operation over the interval of interest. The averaging interval used in this study is $10 \mathrm{~s}$. This value is chosen based on an evaluation of several time intervals ranging from 10 to $60 \mathrm{~s}$ presented in Jiao et al. (2013). In this study, the $S_{4}$ index is calculated using a moving window, which outputs the $S_{4}$ index every second with the averaging window length of $10 \mathrm{~s}$ where the current sample is centered.

The $C / N_{0, \mathrm{SI}}$ is obtained by converting the $\mathrm{SI}_{\text {norm }}$ to an absolute measure of signal-to-noise ratio with the nominal carrierto-noise ratio as the baseline:

$C / N_{0, \mathrm{SI}}=\mathrm{SI}_{\text {norm }}+C / N_{0, \text { nominal }}$

where $C / N_{0, \text { nominal }}$ denotes the nominal carrier-to-noise ratio in $\mathrm{dB}-\mathrm{Hz}$. It should be noted that $C / N_{0, \text { nominal }}$ varies among 
different frequencies and different satellites. In this study, we obtain the $C / N_{0, \text { nominal }}$ values during each scintillation event by examining the corresponding quiet-time $C / N_{0, \text { nominal }}$ immediately before and/or after. As discussed in prior publications (Xu and Morton 2017), we use $C / N_{0, S I}$ instead of $\mathrm{SI}_{\text {norm }}$ to represent amplitude fading levels. This is the case because $\mathrm{SI}_{\text {norm }}$ itself does not convey the signal power level relative to the noise level, and therefore, does not reflect the degree of degradation that signal fading imposes on the tracking loop.

\section{Bit decoding error}

The navigation data bits are directly estimated based on the sign of $I_{\mathrm{p}}$, the prompt correlator output modeled as follows (Ward et al. 2005):

$I_{\mathrm{P}}=A \cdot N \cdot D \frac{\sin (\pi \cdot \Delta f \cdot T)}{\pi \cdot \Delta f \cdot T} \cdot R(\Delta \tau) \cdot \cos (\Delta \phi)+n_{\mathrm{I}}$

where $A$ is signal amplitude; $N$ is the number of samples within the integration time; $D$ is the current message bit $(+1$ or -1$) ; \Delta f$ is the Doppler frequency error in $\mathrm{Hz} ; T$ is the integration time; $\Delta \tau$ is code phase error of prompt replica code in unit of code chips; $R(\cdot)$ is the autocorrelation function of ranging code; $\Delta \phi$ is the carrier phase error in rad; $n_{I}$ is the thermal noise in the I channel.

When the tracking loop reaches steady-state under a nominal signal condition, the signal power should dominate over the noise power and dictate the sign of $I_{\mathrm{p}}$ in (7). In addition, the term $\frac{\sin (\pi \cdot \Delta f \cdot T)}{\pi \cdot \Delta f \cdot T} \cdot R(\Delta \tau)$ is positive. Therefore, the sign of $I_{\mathrm{p}}$ is determined by the sign of the product $D \cdot \cos (\Delta \phi)$.

The bit difference $\Delta D$ is the product of the decoded data bits and a truth reference. In this work, the product from the Bit Grabber Network (bitArc) of Constellation Observing System for Meteorology, Ionosphere, and Climate (COSMIC) (UCAR 2013) is used as the data bit truth, as shown in Fig. 1.

The conventional PIF-based PLL implements the Costas discriminator which is insensitive to the data bit. As $\Delta \phi$ settles down to close to 0 or $\pm \pi, \Delta D$ can output either continuous " 1 " or " -1 " for each data bit depending on the actual steady-state value of $\Delta \phi$. The navigation message decoding will not be affected regardless of the sign of the $\Delta D$ output because the GPS L1 navigation message encoding scheme is designed to overcome this ambiguity. This is enabled during bit encoding by making the polarity of the information bits (the first 24 bits) of a certain word dependent on the 30th bit of the last word: the polarity of the information bits of a certain word is reversed if the polarity of the 30th bit of the last word is " -1 " (US Air Force 2013). This way, the reversion, which is introduced by $\cos (\Delta \phi)$ when $\Delta \phi$ settles at $\pm \pi$ and shared between words, can be removed during message decoding word-by-word. Therefore, the case when $\Delta D$ is continuously being " -1 " should not be considered a decoding error. For more details, please refer to the GPS Interface Control Document (ICD) (US Air Force 2013).

However, during equatorial scintillation, large phase disturbances during signal amplitude fading will affect the bit decoding process by corrupting the relationship between $I_{\mathrm{p}}$ and $D$. As a result, $\Delta D$ may oscillate between " 1 " and "- 1 ", which will prevent correct decoding of the navigation message. After the signal recovered from a fade, $\Delta D$ may settle at a value that is reversed from the one before the fading (from " 1 " to " -1 " or vice versa). Therefore, the number of bit errors is counted as the number of reversals of $\Delta D$ between " 1 " and " -1 " during a fading event.

An example of such $\Delta D$ oscillations during a deep fade (PRN 6, starting time 22:29:16 UTC) is given in Fig. 2.

Figure 2 illustrates that the fading duration definition and the associated data bit decoding error. The fading duration is the time interval between the instance where $C / N_{0, \mathrm{SI}}$ drops below a threshold value $C / N_{0, \text { threshold }}$ and the instance where $C / N_{0, \mathrm{SI}}$ rises from the fading and settles above the $C / N_{0, \text { threshold }}$. During the fading duration, both algorithms produced oscillations on the $\Delta D$ results. Both $\Delta D$ results indicate five occurrences of bit reversions.

It should be mentioned that an alternative bit decoding approach is the differential bit decoding method described in Van Dierendonck (1996) for a frequency lock loop (FLL). This approach detects the bit sign changes by examining the signs of the dot-products of the I and Q correlator outputs from two consecutive epochs. It is applicable to receivers utilizing FLL as it does not require the lock of carrier phase and is, therefore, suitable for applications on platforms

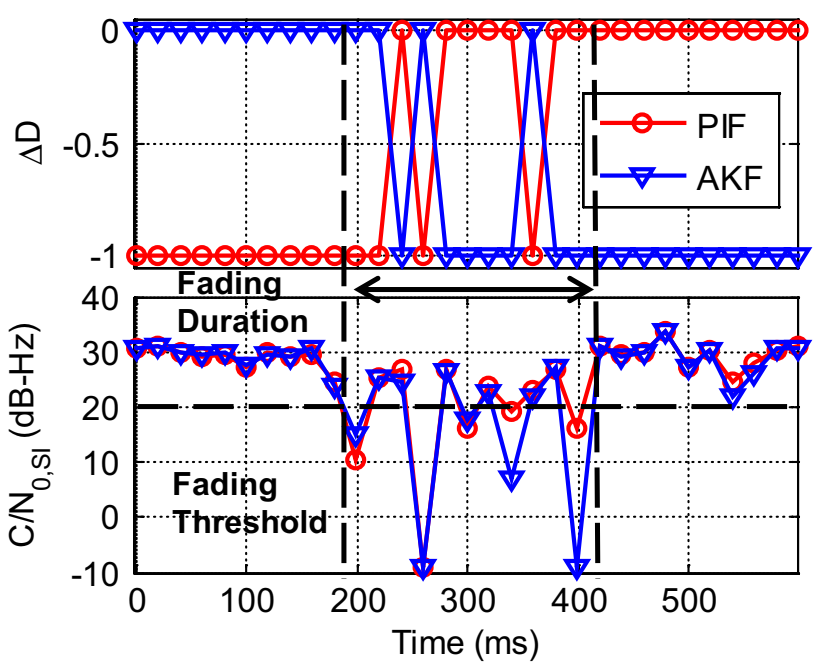

Fig. 2 BDE example processed using PIF and AKF-based algorithms on GPS PRN 6 L1 signal at Ascension Island, starting at 22:29:16 UTC 
undergoing high dynamics. However, the strong equatorial scintillation scenario discussed in this study is characterized with both fast phase changes (which has an equivalent effect of high signal dynamics) and concurrent signal deep fading. Application of FLL to the data set indicated that it encountered difficulties maintaining lock to the signal, and therefore, prevented further assessment of the differential bit decoding performance.

\section{Real scintillation data BDE processing results and statistical analysis}

This section begins by briefly describing the scintillation data collection system and the IF data used in this study. Then the processing results from almost $14 \mathrm{~h}$ of scintillation data on six GPS satellites are presented to establish statistical correlations between the BDE occurrences and various amplitude scintillation indicators (average $S_{4}$ levels, fading occurrence frequency, fading depths and durations). Finally, to assess the improvement of the advanced AKF-based PLL over the conventional PLL on bit decoding performance, the BDE results of the two carrier tracking algorithms during a segment of the scintillation periods are compared.

\section{Scintillation data collection system}

The IF data used in this study were collected during an experimental campaign on Ascension Island in March 2013. Figure 3 depicts the reconfigurable multi-GNSS SDR data collection system used in this campaign, which contains five $\mathrm{RF}$ front-ends each configured to collect signals within one frequency band: GPS L1/Galileo E1, GPS L2C, GPSL5/ Galileo E5a, GLONASS L1, and BeiDou B1 signals (Peng and Morton 2011). All front-ends share the same wideband antenna input and are driven by the same low phase noise OCXO with zero IF and 4-bit resolution. The front-ends collected data for $5 \mathrm{~h}$ (20:00-01:00 UTC) on March 7-10, resulting in a total of $20 \mathrm{~h}$ of IF data.

\section{Statistical summary on the BDE occurrences and amplitude scintillation and fading characteristics}

In this work, a total of about $14 \mathrm{~h}$ of GPS L1 IF scintillation data with $S_{4}>0.5$ from six satellites are processed using the PIF-PLL. The BDE results obtained from the data are used for correlative analysis with amplitude scintillation and fading indicators. In addition, a total of $4 \mathrm{~h}$ of IF data from March 7 are further processed using AKF-PLL to assess its improvement on the bit decoding performance over the PIF-PLL implementation. Table 1 summarizes the durations, average $S_{4}$ indices, the number of fades and fading

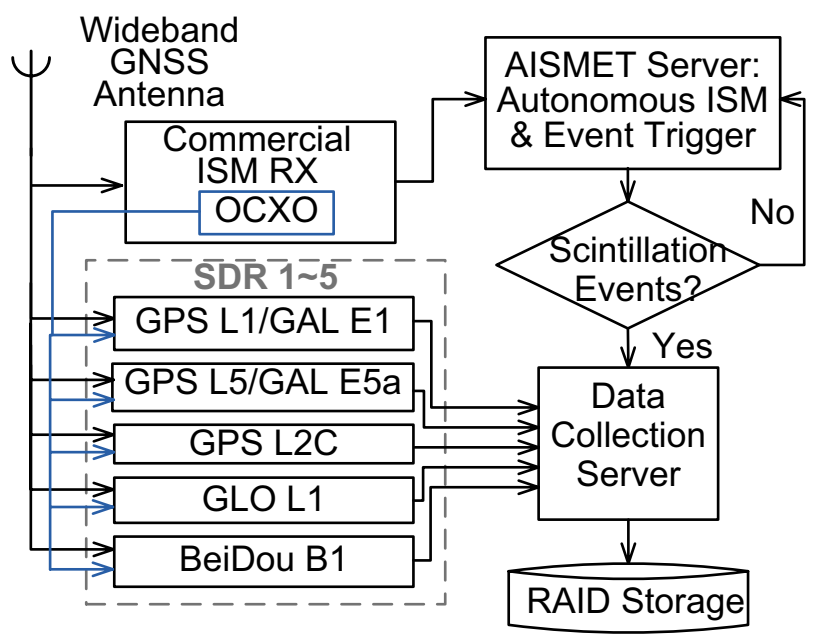

Fig. 3 Schematic diagram of the wideband reconfigurable multiGNSS data collection system setup at Ascension Island on March 7-10, 2013 (Xu and Morton 2017)

frequency of occurrences (per minute), the number of BDE occurrences (in bits) and its frequency of occurrence (per minute) for each scintillation event.

The following subsections present the statistical results derived from Table 1.

BDE occurrence frequency dependence on average $S_{4}$ value and fading occurrence frequency

A total of 3720 bits of BDE occurred during $13.8 \mathrm{~h}$ of the scintillation periods, resulting in an average frequency of occurrence of around 4.5 bits per minute. Figure 4 plots the number of fades per minute and average $S_{4}$ values listed in Table 1 and clearly showed nearly linear correlations between BDE frequency of occurrence with the average S4 values and the average number of fades.

\section{Percentage of BDE occurrences with respect to $S_{4}$ levels}

To examine the correlation between the occurrence of BDE and amplitude scintillation levels on a finer temporal scale, the $S_{4}$ estimations [obtained every second, as described after (5)] were categorized into three segments shown in Table 2. Also listed in Table 2 are the number and percentage of the $S_{4}$ samples for each segment and corresponding BDE occurred during each segment. The probability of BDE occurrence listed in the rightmost column is obtained by dividing the number of $S_{4}$ samples that experience BDE by the total number of $S_{4}$ samples within the segment. Table 2 indicates that during the selected scintillation durations the probability of BDE occurrence increases as the amplitude scintillation intensity increases. 
Table 1 Summary of the number of BDE and the amplitude scintillation parameters during the selected scintillation periods in the Ascension Island data

\begin{tabular}{|c|c|c|c|c|c|c|c|}
\hline Date & PRN & $\begin{array}{l}\text { Event } \\
\text { duration } \\
\text { (h) }\end{array}$ & Avg. $S_{4}$ & \# of fades & \# of fades/min & \# of BDE (bit) & \# of $\mathrm{BDE} / \mathrm{min}$ \\
\hline \multirow[t]{4}{*}{$3 / 7$} & 6 & 0.94 & 0.59 & 177 & 3.13 & 436 & 7.73 \\
\hline & 14 & 1.12 & 0.49 & 121 & 1.80 & 69 & 1.03 \\
\hline & 22 & 1.09 & 0.41 & 23 & 0.35 & 22 & 0.34 \\
\hline & 29 & 0.83 & 0.45 & 7 & 0.14 & 7 & 0.14 \\
\hline \multirow[t]{5}{*}{$3 / 8$} & 6 & 0.33 & 0.60 & 46 & 2.32 & 99 & 5.00 \\
\hline & 14 & 0.22 & 0.57 & 8 & 0.61 & 15 & 1.14 \\
\hline & 24 & 0.85 & 0.73 & 373 & 7.3 & 744 & 14.6 \\
\hline & 29 & 0.98 & 0.65 & 228 & 3.88 & 494 & 8.40 \\
\hline & 31 & 0.35 & 0.89 & 141 & 6.71 & 320 & 15.2 \\
\hline \multirow[t]{3}{*}{$3 / 9$} & 6 & 1.31 & 0.56 & 75 & 0.95 & 118 & 1.50 \\
\hline & 29 & 0.41 & 0.78 & 85 & 3.46 & 162 & 6.59 \\
\hline & 31 & 0.53 & 0.45 & 3 & 0.09 & 7 & 0.22 \\
\hline \multirow[t]{4}{*}{$3 / 10$} & 6 & 1.74 & 0.57 & 223 & 2.14 & 429 & 4.11 \\
\hline & 14 & 0.93 & 0.53 & 17 & 0.30 & 36 & 0.65 \\
\hline & 24 & 1.04 & 0.53 & 184 & 2.95 & 233 & 3.73 \\
\hline & 29 & 1.14 & 0.54 & 189 & 2.76 & 529 & 7.73 \\
\hline Sum & & 13.81 & & 1900 & & 3720 & 4.49 \\
\hline
\end{tabular}

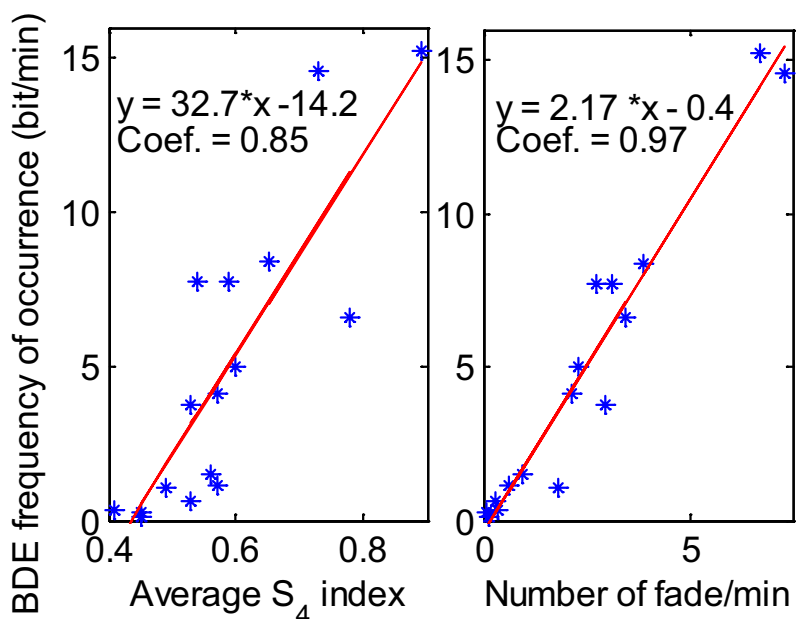

Fig. 4 Relationships between the BDE frequency of occurrence and the average $S_{4}$ results (left panel) and the number of fades per minute (right panel) derived from the processing results of $14 \mathrm{~h}$ of scintillation data

Table 2 Number and percentage of the $S_{4}$ estimate samples and of the $\mathrm{BDE}$, and the probability of BDE occurrence under different $S_{4}$ levels

\begin{tabular}{llll}
\hline$S_{4}$ level & \# of $S_{4}$ samples/\% & \# of BDE/\% & $\begin{array}{l}\text { Prob- } \\
\text { ability of } \\
\text { occur. }\end{array}$ \\
\hline$<0.7$ & $36,700 / 74$ & $667 / 18$ & 0.01 \\
$0.7-0.9$ & $7985 / 16$ & $1326 / 36$ & 0.05 \\
$>0.9$ & $4887 / 10$ & $1727 / 46$ & 0.12 \\
\hline
\end{tabular}

Table 3 Number and percentage of the fades and of the BDE, and the probability of occurrence under different fading levels

\begin{tabular}{llll}
\hline $\begin{array}{l}\text { Minimum } C / N_{0, \mathrm{SI}} \\
(\mathrm{dB}-\mathrm{Hz})\end{array}$ & \# of fades/\% & \# of BDE/\% & $\begin{array}{l}\text { Probability } \\
\text { of occur. }\end{array}$ \\
\hline $15-20$ & $224 / 12$ & $31 / 0.8$ & 0.06 \\
$10-15$ & $163 / 9$ & $10 / 0.3$ & 0.03 \\
$5-10$ & $644 / 34$ & $266 / 7$ & 0.15 \\
$<5$ & $869 / 45$ & $3413 / 91.9$ & 0.81 \\
\hline
\end{tabular}

Percentage of BDE occurrences with respect to fading levels

The fading depth is determined by the minimum $C / N_{0, \mathrm{SI}}$ value during the fading duration. Table 3 summarizes the number and corresponding percentage of the fades under different fading depth ranges and those of the BDE concurrent with these fades. The probability of BDE occurrence listed in the rightmost column is obtained by dividing the number of fades that are associated with BDE occurrences by the total number of fades within each fading depth range. Table 3 shows that the majority of BDE occurred during fades with extremely deep fading of below $5 \mathrm{~dB}-\mathrm{Hz}$ with a probability of occurrence up to 0.81 , while these extremely deep fades comprise $45 \%$ of the total number of fades. 


\section{Correlation between BDE occurrence and extremely deep fade duration}

Based on the number of BDE occurred during each extremely deep fade $\left(C / N_{0, S I}<5 \mathrm{~B}-\mathrm{Hz}\right)$, these fades are divided into five groups with the corresponding percentages plotted in Fig. 5. It shows that the larger number of BDE within a fade is much less likely compared to the smaller number of BDE within a fade. More than $70 \%$ of the cases involve less than five BDE occurrences within one fade. The mean number of BDE occurred during one fade is 4.9 bits for these extremely deep fades.

The correlation between the number of BDE occurred during a certain fade and the duration of the corresponding fade is plotted in Fig. 6. The data show that the number of BDE during one fade grows almost linearly with the duration of the concurrent fade, with a ratio of 8.5 (bit per second) and a correlation coefficient of 0.7 .

\section{BDE improvement using AKF-PLL}

The BDE results obtained from the selected scintillation data of March 7, 2013 provide a glimpse of the performance improvement of the AKF-PLL over PIF-PLL. Table 4 lists the number of BDE occurrences during each event in the dataset obtained using the two PLL implementations. It shows that the AKF overall outperformed the PIF in generating less decoding error by $17 \%$. The majority of $\mathrm{BDE}$ occurred on PRN 6, where AKF-PLL showed the best improvement over PIF-PLL in reducing the number of BDE by $20 \%$. This finding suggests that by utilizing advanced tracking algorithms such as adaptive architectures and modeling of the scintillation effect, the decoding performance can be considerably improved.



Fig. 5 Distribution of the extremely deep fades $\left(C / N_{0, \mathrm{SI}}<5 \mathrm{~B}-\mathrm{Hz}\right)$ with respect to the number of BDE occurred during each of them

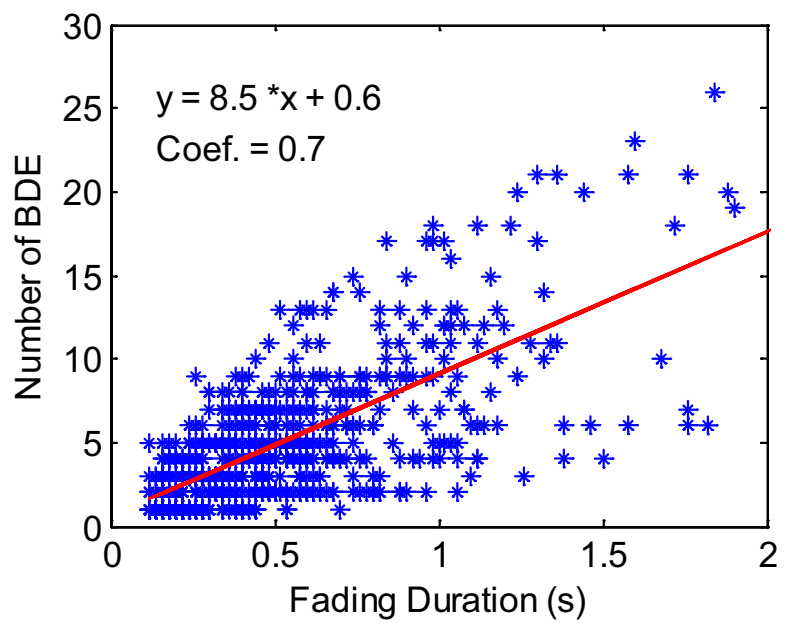

Fig. 6 Relationship between the number of BDE occurred during an extremely deep fade and the corresponding fading duration

\section{Summary}

We analyzed the correlation between BDE occurrences and amplitude scintillation and fading levels based on processing results from 14-h strong scintillation IF data collected on Ascension Island in March 2010. A conventional thirdorder PIF-PLL is used to process the data and the COSMIC data bit archives are used as a truth reference to identify BDE occurrences. A total of 3720 bits of BDE were identified, indicating an overall rate of occurrence of 4.5 bits per minute. The results also indicated that the number of BDE occurrence is linearly related to the average $S_{4}$ index and the number of fades per minute.

The number of BDE occurrence is further mapped to three segments of the $S_{4}$ estimation values obtained every second. The probability of BDE occurrence clearly showed the BDE occurrence is higher for the higher S4 value segment.

The minimum $C / N_{0, \mathrm{SI}}$ value during the fades was utilized to quantify the fading depths and to correlate with the occurrence of BDE. Our data analysis shows that up to $92 \%$ of the BDE occurred during extremely deep fades with minimum $C / N_{0, \mathrm{SI}}$ below $5 \mathrm{~dB}-\mathrm{Hz}$, which comprise $45 \%$ of the total fades. The extremely deep fades were then used to derive the

Table 4 Summary of number of BDE occurrences based on the processing results obtained from the scintillation data selected on March 7, using the conventional (PIF) and the advanced AKFbased PLL algorithm

\begin{tabular}{lrr}
\hline PRN\# & PIF & AKF \\
\hline 6 & 436 & 352 \\
14 & 69 & 60 \\
22 & 22 & 22 \\
29 & 7 & 6 \\
Sum & 534 & 440 \\
\hline
\end{tabular}


relationship between the number of BDE occurring during one fade and the duration of the fade. The results show that most of these fades contain around 5 bits of BDE, while the maximum number of BDE during one fade can exceed 25 bits. A linear relationship was obtained with a frequency of 8.5 bits of BDE per second during extremely deep fading and a correlation coefficient of 0.7 .

In addition, an advanced AKF-based PLL was also used to process a subset of the selected scintillation data. Comparison of the resulting BDE indicates that the AKF-based tracking loop has better performance over the conventional PLL in reducing the occurrences of BDE by $17 \%$.

Acknowledgements The Ascension Island data were collected with support by Dr. Todd Pedersen from the Air Force Research Laboratory (AFRL) at Kirkland Air Force Base. The equipment used in the data collection system was funded by a Grant through AFRL (FA865008-D-1451), Wright Patterson Air Force Base. Steven Taylor and Harrison Bourne from the Satellite Navigation and Sensing Lab at the University of Colorado developed the data collection system. Mr. Dongyang Xu's work is funded by Colorado State University and a NASA Grant (NNX15AT54G).

Open Access This article is distributed under the terms of the Creative Commons Attribution 4.0 International License (http://creativeco mmons.org/licenses/by/4.0/), which permits unrestricted use, distribution, and reproduction in any medium, provided you give appropriate credit to the original author(s) and the source, provide a link to the Creative Commons license, and indicate if changes were made.

\section{References}

Aquino M, Moore T, Dodson A, Waugh S, Souter J, Rodrigues FS (2005) Implications of ionospheric scintillation for GNSS users in Northern Europe. J Navig 58(2):241-256

Basu S, Grove KM, Basu S, Sultana PJ (2002) Specification and forecasting of scintillations in communication and navigation links: current status and future plans. J Atmos Solar Terr Phys 64(16):1745-1754. https://doi.org/10.1016/S1364-6826(02)00124 $-4$

Buchert S, Zangerl F, Sust M, André M, Eriksson A, Wahlund J, Opgenoorth H (2015) SWARM observations of equatorial electron densities and topside GPS track losses. Geophys Res Lett 42(7):2088-2092

Carroll M, Morton Y (2014) Triple frequency GPS signal tracking during strong ionospheric scintillations over Ascension Island. In: Proceedings of IEEE/ION PLANS 2014, Institute of Navigation, Monterey, CA, USA, May 5-8, pp 43-49

Conker RS, El-Arini MB, Hegarty C, Hsiao T (2003) Modelling the effects of ionospheric scintillation on GPS/SBAS availability. Radio Sci 38(1):1-23

Jiao Y, Morton YT, Taylor S, Pelgrum W (2013) Characterization of high latitude ionospheric scintillation of GPS signals. Radio Sci 48(6):698-708

Jiao Y, Xu D, Morton Y, Rino C (2016) Equatorial scintillation amplitude fading characteristics across the GPS frequency bands. Navigation 63(3):267-281

Kintner PM, Ledvina BM, de Paula ER (2007) GPS and ionospheric scintillations. Space Weather. https://doi.org/10.1029/2006S W000260
Lee J, Morton Y, Lee J, Moon H-S, Seo J (2017) Monitoring and mitigation of ionospheric anomalies for GNSS-based safety critical systems: a review of up-to-date signal processing techniques. Special issue on Advances in Signal Processing for Global Navigation Satellite Systems. IEEE Signal Process Mag 34(5):96-110

Morton R (2014) Investigation of the impact of solar storms on the Global Positioning System receivers at high latitudes. In: Proceedings of ION ITM 2014, Institute of Navigation, San Diego, CA, USA, January 27-29, pp 700-708

Morton Y, Jiao Y, van Graas F, Vinande E, Pujara N (2015a) Analysis of receiver multi-frequency response to ionospheric scintillation in Ascension Island, Hong Kong, and Singapore. In: Proceedings of ION PNT 2015, Institute of Navigation, Honolulu, HI, USA, April 20-23, pp 10-16

Morton Y, Jiao Y, Taylor S (2015b) High-latitude and equatorial ionospheric scintillation based on an event-driven multi-GNSS data collection system. In: Proceedings of ionospheric effects symposium 2015, Alexandria, VA, USA, May 12-14. http:// ies2015.bc.edu/wp-content/uploads/2015/05/127-Morton-Paper .pdf

Myer G, Morton Y (2018) Ionosphere scintillation effects on GPS measurements, a new carrier-smoothing technique, and positioning algorithms to improve accuracy. In: Proceedings of ION ITM 2018, Institute of Navigation, Reston, VA, USA, January 29-311, pp 420-439

Oliveira K, Moraes A, Costa E, Muella M, de Paula ER, Perrella W (2014) Validation of the alpha-mu model of the power spectral density of GPS ionospheric amplitude scintillation. Int J Antennas Propag. https://doi.org/10.1155/2014/573615

Peng S, Morton Y (2011) A USRP2-Based multi-constellation and multi-frequency GNSS software receiver for ionosphere scintillation studies. In: Proceedings of ION ITM 2011, Institute of Navigation, San Diego, CA, January 24-26, pp 1033-1042

Psiaki M, Humphreys T, Cerruti A, Powell S, Kintner P (2007) Tracking L1 C/A and L2C signals through ionospheric scintillations. In: Proceedings of ION GNSS 2007, Institute of Navigation, Fort Worth, TX, USA, September 25-28, pp 246-268

Seo J, Walter T, Chiou TY, Enge P (2009) Characteristics of deep GPS signal fading due to ionospheric scintillation for aviation receiver design. Radio Sci. https://doi.org/10.1029/2008RS004077

Seo J, Walter T, Enge P (2011) Correlation of GPS signal fades due to ionospheric scintillation for aviation applications. Adv Sp Res 47(10):1777-1788

Tsui J (2005) Fundamentals of global positioning system receivers: a software approach, 2nd ed. Wiley, Oxford. https://doi. org/10.1002/0471200549

UCAR (2013) GPS data bits hourly archive files. http://cosmic-io. cosmic.ucar.edu/cdaac/login/fidrt/level1a/bitArc/

US Air Force (2013) Interface specification IS-GPS-200H. NAVSTAR GPS Space Segment/Navigation User Interfaces

Van Dierendonck AJ, Klobuchar JA, Hua Q (1993) Ionospheric scintillation monitoring using commercial single frequency C/A code receivers. In: Proceedings of ION ITM 1993, Institute of Navigation, Salt Lake City, UT, USA, September 22-24, pp 1333-1342

Van Dierendonck AJ (1996) GPS receivers, Chap. 8. In: Parkinson BW et al. (ed) Global positioning system: theory and applications, vol 1. American Institute of Aeronautics and Astronautics, Washington, DC

Ward PW, Betz JW, Hegarty CJ (2005) GPS satellite signal acquisition and tracking. In: Kaplan ED, Hegarty CJ (eds) Understanding GPS: principles and applications, 2nd edn. Artech House, MA, pp $153-240$

Xiong C, Stolle C, Lühr H (2016) The Swarm satellite loss of GPS signal and its relation to ionospheric plasma irregularities. Sp Weather 14(8):563-577 
Xu D, Morton Y (2017) A semi-open loop GNSS carrier tracking algorithm for monitoring strong equatorial scintillation. IEEE Trans Aerosp Electron Syst 54(2):722-738

Xu D, Morton Y, Taylor S (2014) Algorithms and results of tracking BeiDou signals during strong ionospheric scintillation over Ascension Island. In: Proceedings of ION ITM 2014, Institute of Navigation, San Diego, CA, USA, January 27-29, pp 730-735

Xu D, Morton Y, Jiao Y, Rino C (2017) Robust GPS carrier tracking algorithms during strong equatorial scintillation for dynamic platforms. In: Proceedings of ION GNSS + 2017, Institute of Navigation, Portland, OR, USA, September 25-29, pp 4112-4121

Yang R, Ling KV, Poh EK, Morton Y (2017a) Generalized GNSS signal carrier tracking: part I-modelling and analysis. IEEE Trans Aerosp Electron Syst 53(4):1781-1797

Yang R, Morton Y, Ling K, Poh E (2017b) Generalized GNSS signal carrier tracking - part II: optimization and implementation. IEEE Trans Aerosp Electron Syst 53(4):1798-1811

Yeh CK, Liu CH (1982) Radio wave scintillations in the ionosphere. Proc IEEE 70(4):324-360

Zhang L, Morton Y, Zhou Q, van Graas F, Beach T (2010) Characterization of GNSS signal parameters under ionosphere scintillation conditions using software-based tracking algorithms. In: Proceedings of IEEE/ION PLANS 2010, Indian Wells, CA, USA, May 4-6, pp 264-275

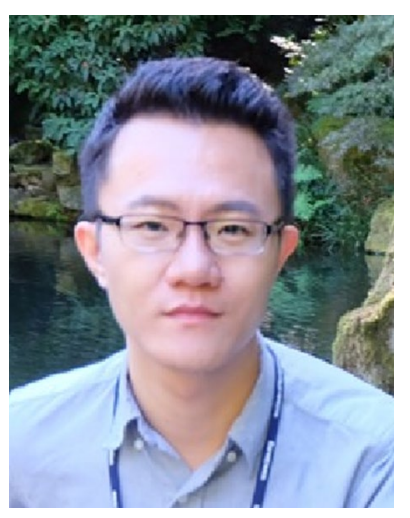

Dongyang $\mathrm{Xu}$ is a $\mathrm{Ph} . \mathrm{D}$. student in the Department of Electrical and Computer Engineering at Colorado State University. He received a B.S. in Electrical Engineering from China Agricultural University in 2009, an M.S. in Electrical Engineering from Beihang University, China in 2012 and a second M.S. in Computational Engineering and Science from Miami University, Ohio in 2014. His research interests include GNSS software receiver tracking algorithm development and performance analysis under ionospheric scintillation.

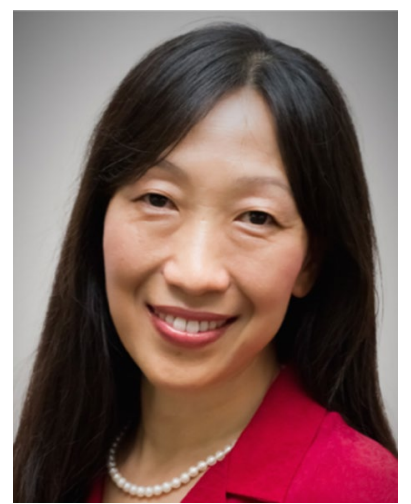

Y. T. Jade Morton is a Professor at the Smead Aerospace Engineering Sciences Department and the head of the Satellite Navigation and Sensing Lab at the University of Colorado (CU), Boulder. She received a Ph.D. in Electrical Engineering (EE) from Penn State, a MS in EE from Case Western Reserve University, and a BS in Physics from Nanjing University in China. Prior to joining CU in 2017, she was an electrical engineering professor at Colorado State University and Miami University, and a post-doctoral fellow at the University of Michigan. Her research interests lie at the intersection of satellite navigation technologies and remote sensing of the ionosphere, troposphere, and surface. She is a recipient of Institute of Navigation (ION) Thurlow and Burka award, and a fellow of IEEE and ION. 